\title{
Fusion Image Guidance for Supra-Aortic Vessel Catheterization in Neurointerventions: A Feasibility Study
}

\author{
(D) A. Feddal, (D) S. Escalard, (D). Delvoye, (D). Fahed, (D).P. Desilles, (D) K. Zuber, (DH. Redjem, (D).S. Savatovsky, (D) G. Ciccio,
} (i) S. Smajda, (i) M. Ben Maacha, (D) M. Mazighi, (D) M. Piotin, and (D) Rlanc

\begin{abstract}
BACKGROUND AND PURPOSE: Endovascular navigation through tortuous vessels can be complex. Tools that can optimise this access phase need to be developed. Our aim was to evaluate the feasibility of supra-aortic vessel catheterization guidance by means of live fluoroscopy fusion with MR angiography or CT angiography.
\end{abstract}

MATERIALS AND METHODS: Twenty-five patients underwent preinterventional diagnostic MRA, and 8 patients underwent CTA. Fusion guidance was evaluated in 35 sessions of catheterization, targeting a total of 151 supra-aortic vessels. The time for MRA/CTA segmentation and fluoroscopy with MRA/CTA coregistration was recorded. The feasibility of fusion guidance was evaluated by recording the catheterizations executed by interventional neuroradiologists according to a standard technique under fluoroscopy and conventional road-mapping independent of the fusion guidance. Precision of the fusion roadmap was evaluated by measuring (on a semiquantitative 3-point scale) the maximum offset between the position of the guidewires/catheters and the vasculature on the virtual CTA/MRA images. The targeted vessels were divided in 2 groups according to their position from the level of the aortic arch.

RESULTS: The average time needed for segmentation and image coregistration was $7 \pm 2$ minutes. The MRA/CTA virtual roadmap overlaid on live fluoroscopy was considered accurate in $84.8 \%$ (128/151) of the assessed landmarks, with a higher accuracy for the group of vessels closer to the aortic arch (92.4\%; OR, 4.88; 95\% Cl, 1.83-11.66; $P=.003$ ).

CONCLUSIONS: Fluoroscopy with MRA/CTA fusion guidance for supra-aortic vessel interventions is feasible. Further improvements of the technique to increase accuracy at the cervical level and further studies are needed for assessing the procedural time savings and decreasing the $\mathrm{x}$-ray radiation exposure.

W ith the increasing use of endovascular treatments for cerebrovascular diseases, in an aging population, physicians are more frequently facing complex endovascular navigation. Limitations to the navigation of endovascular devices from the aortic arch to the intracranial vessels still remain, ${ }^{1,2}$ and the speed and safety of access through the vascular tortuosity need to be enhanced. $^{3}$ Access through tortuous vessels can make the treatment complex and leads to increased procedural time, $\mathrm{x}$-ray exposure, and rate of adverse events or failure. , $^{2,-6}$

Routine supra-aortic vessel catheterization in neurovascular procedures uses conventional road-mapping (superimposition of contrast-filled vessels on fluoroscopy using digital subtraction). It

Received March 13, 2020; accepted after revision June 3.

From the Interventional Neuroradiology Unit (A.F., S.E., F.D., R.F., J.P.D., K.Z., H.R., G.C., S.S., M.B.M., M.M., M.P., R.B.) and Diagnostic Neuroradiology Unit (J.S.S.), Fondation Ophtalmologique Adolphe de Rothschild, Paris, France; Université Paris Denis Diderot (J.P.D., M.M., M.P., R.B.), Sorbonne Paris Cite, Paris, France; Laboratory of Vascular Translational Science (J.P.D., M.M., M.P., R.B.), U1148 Institut National de la Santé et de la Recherche Médicale, Paris, France.

Please address correspondence to Raphaël Blanc, MD, MSc, Department of Interventional Neuroradiology, Fondation Ophtamologique A de Rothschild, 29 Rue Manin, 75019 Paris, France; e-mail: rblanc@for.paris

http://dx.doi.org/10.3174/ajnr.A6707

provides good spatial and contrast resolution and enhances direct catheterization, which can cause vasospasm, dissection, and thromboembolic events due to wire and guide traumatic manipulation and atheromatous plaque dislodgement.

However, its main limitation is inherent in its static projection. Because tortuous anatomy is more frequently encountered in clinical practice, physicians require different working projections, varying the $\mathrm{C}$-arm detector angle to clear an ostium or delineate loops. This requirement leads to extra contrast administration each time a new roadmap is generated.

Live 3D road-mapping techniques allow dynamic vessel roadmapping, with live adaptation to the $\mathrm{C}$-arm movements. This technique combines real-time fluoroscopy with a previously performed CTA or MRA and has gained wide acceptance for aortic or peripheral endovascular procedures. It is claimed and now widely recognized that it could enhance catheterization abilities and reduce extra contrast administration and procedural time, especially in complex vascular catheterizations. ${ }^{7-10}$

The use of such fusion imaging techniques is also gaining acceptance in the interventional neuroradiology field for intracranial use, ${ }^{11-13}$ especially for primary access (navigation of the 
guiding catheter or long introducer sheath) either through venous ${ }^{14}$ or arterial approaches. ${ }^{15}$

The goal of our study was to assess the feasibility and evaluate the spatial accuracy of aortic arch and supra-aortic cervical vessel catheterization by means of fusion guidance using VesselNavigator software (Philips Healthcare).

\section{MATERIALS AND METHODS}

This study is a single-center (Fondation Ophtalmologique Adolphe de Rothschild, Paris, France) prospective study designed to evaluate the feasibility of aortic arch and supra-aortic cervical vessel catheterization under fusion guidance in neurointerventional diagnostic or therapeutic angiograms. VesselNavigator software has a CE Mark but was used off-label for this study.

The patients were offered participation in the study if they had a confirmed indication for either a diagnostic or therapeutic angiography for any cerebrovascular condition and had undergone noninvasive encephalic and aortic arch vessel MRA or CTA as a part of their diagnostic management. No additional encephalic or aortic arch vessel imaging was based for the specific purpose of the study. No selection was based on age, atherosclerosis status, or any MRA or CTA features predicting a difficult catheterization (vascular tortuosity, anatomic variants).

Oral and written information about fusion guidance was delivered during a medical consultation before the procedure. The study was approved by the institutional ethics committee, and written consent was waived. This study is registered under clinicaltrial.org identifier NCT03269734.

\section{Preprocedural Noninvasive Vascular Imaging}

MRA. Patients were scanned before the procedure on a $3 T$ MR imaging system (Ingenia 3T; Philips Healthcare) using a 16-channel head coil. All examinations included a contrast-enhanced MR angiography of the aortic arch, supra-aortic trunks, and intracranial vessels using the following parameters: coronal plane covering 250 slices, active $\mathrm{TR} / \mathrm{TE}=5.5 / 2.2 \mathrm{~ms}$, flip angle $=27^{\circ}$, number of excitations $=1$, acquisition bandwidth $=476.7 \mathrm{~Hz}, \mathrm{FOV}=380 \times 321$, voxel size $=0.5 \times 0.5 \times 1 \mathrm{~mm}$ (interpolation $=0.495 \times 0.495 \times$ $0.5 \mathrm{~mm}$ ). The imaging time was 1 minute 9 seconds. A bolus-tracking injection technique was used to time the start of data acquisition.

CTA. Patients were scanned (Brilliance CT 64, Philips Healthcare) in a caudocranial fashion from the aortic arch to the circle of Willis (vertex). A bolus injection of contrast was given according to the patient's weight (usually $60-80 \mathrm{~mL}$ ) through an intravenous access (18- or 20-ga catheter, antecubital fossa) at a rate of 3-5 mL per second with a $30-\mathrm{mL}$ saline chaser bolus after contrast injection. An automatic bolus-tracking technique was used with an ROI in the ascending thoracic aorta. Data were acquired when the threshold of $110 \mathrm{HU}$ was reached with a minimum delay of 4 seconds. The technical parameters of our acquisition protocol were the following: collimation $=64 \times 0.6 \mathrm{~mm}$, gantry rotation $=330 \mathrm{~ms}$, tube voltage $=120 \mathrm{kV}$ (peak). The overall acquisition time $(250 \mathrm{~mm})$ was around 4 seconds. The $0.6-\mathrm{mm}$ thickness images were reconstructed at $0.4 \mathrm{~mm}$ in the axial plane and sent to the PACS and used for dedicated MPRs, MIPs, and 3D rendering in the postprocessing workstation.
Anesthesia Protocol. At our institution, all procedures are performed with the patient under the supervision of an anesthesiologist; scheduled patients (excluding emergencies) have dedicated consultation with an anesthesiologist before the intervention. Diagnostic or follow-up angiograms are obtained with the patient under conscious sedation using remifentanil, in addition to local anesthesia for vascular puncture. Embolization procedures (intracranial aneurysms, arteriovenous fistulas, and arteriovenous malformations) are performed with the patient under general anesthesia. Mechanical thrombectomies are usually performed with the patient under conscious sedation.

\section{Procedural Image Fusion and Dynamic 3D Roadmap}

Step 1: Preprocedural Vessel Segmentation. MRA/CTA tridimensional DICOM datasets were imported into the Xtravision workstation and loaded in the VesselNavigator application before the patient was admitted to the suite.

Using a semiautomatic segmentation algorithm incorporated in the software, either the first or the second operator extracted the aortic arch and supra-aortic vessels from the soft tissues.

We defined 9 relevant ROIs to assess the accuracy of the fusion, located $1 \mathrm{~cm}$ above the ostia of the brachiocephalic artery trunk, the right and left common carotid arteries, the right and left internal carotid arteries, the right and left subclavian arteries, and the right and left vertebral arteries. Landmarks were placed on those 9 ROIs, using dedicated circular labels in the VesselNavigator application (Fig 1).

The time needed to complete segmentation and landmark labeling was monitored.

During this step, the operator could define optimal working projections to be used by rotating the segmented vessels. Specific projection angles were stored and were recalled during catheterization. The operator could identify the catheter most adapted to the arterial tree anatomy.

Step 2: 2D-3D Coregistration. The patient was admitted in the operating room and positioned supine on the angiography table. His or her head was set in a neutral position, using head-andshoulder supports. Additional restraining straps were added in case of general anesthesia to immobilize the head in a specific position when necessary.

Image fusion was performed using a $2 \mathrm{D}-3 \mathrm{D}$ alignment: The patient's thorax was positioned at the isocenter of the image $(\mathrm{FOV}=48 \mathrm{~cm})$. The first operator acquired 2 short fluoroscopy runs with the anterior-posterior $\mathrm{C}$-arm, first in right anterior oblique view, then a second short run orthogonal to the previous view.

The segmented 3D volume dataset was merged with fluoroscopy images, matching anatomic features (heart shadow, aortic knob, pulmonary arteries, bones structures) either on the Xtravision workstation outside the operating suite or at tableside using the digital screen with a sterile plastic cover. $3 \mathrm{D}$ roadmap opacity and contrast were adjusted to the first operator's convenience.

Step 3: Perioperative Image Fusion: 3D Dynamic Roadmap. Once the coregistration was set, the VesselNavigator application allowed augmented navigation under 3D dynamic roadmap guidance with 


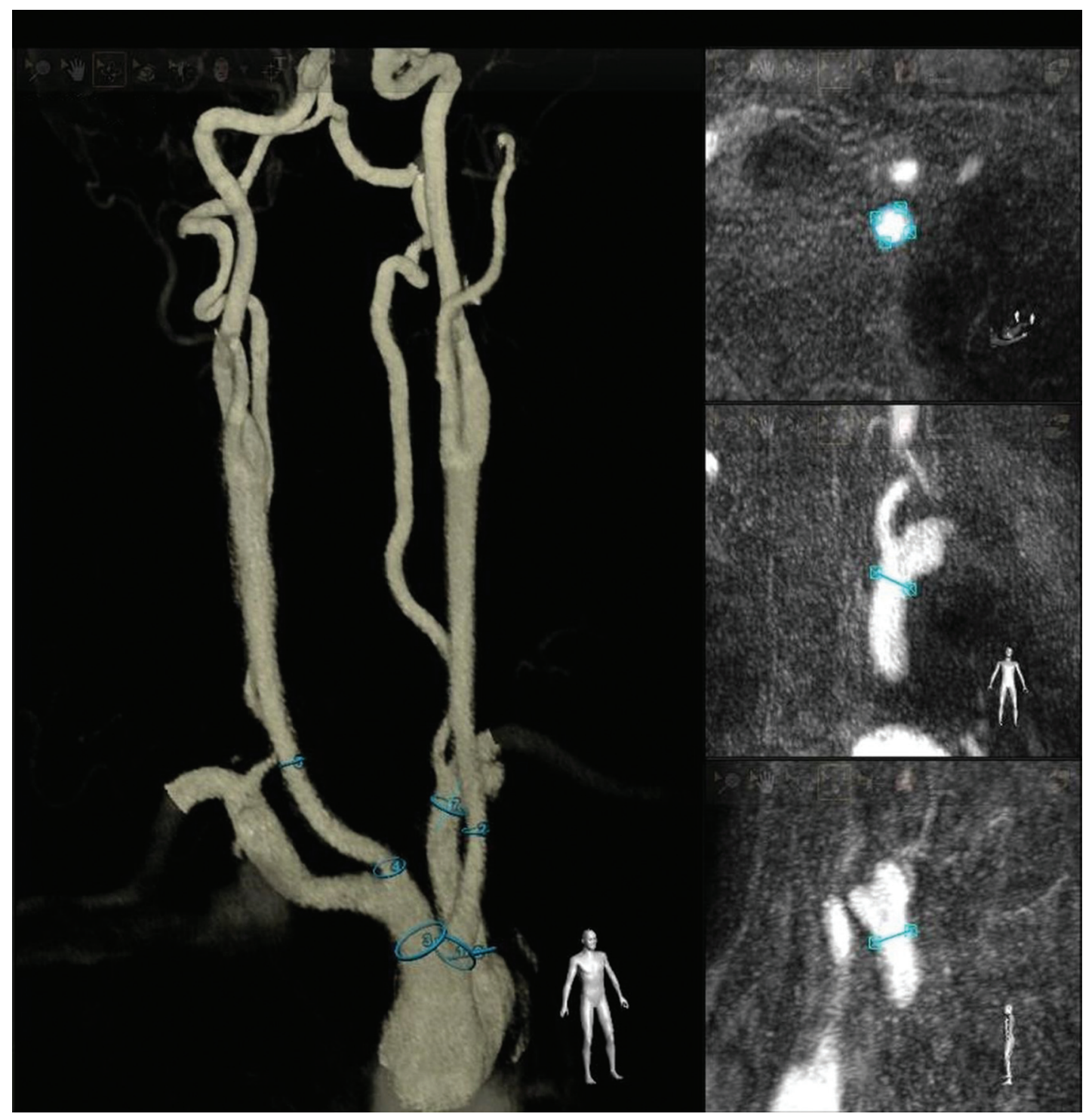

FIG 1. Example of a virtual roadmap of supra-aortic vessels. The vasculature is segmented from the MRA dataset with VesselNavigator software, with landmarks added on the brachiocephalic artery trunk, the right and left common carotid arteries, and the left subclavian artery. During this step, the operator saves projection angles, which can be recalled during catheterization.

the frontal C-arm. The image fusion was real-time, regardless of the C-arm position, magnification, or table manipulation. The first operator performed primary access by navigation of the catheter (diagnostic $5 \mathrm{~F}$ or $6 \mathrm{~F}$ guiding catheter or long introducer sheath) and guidewire from a femoral or radial access to cervical target positions. The operator was able to use, at his or her convenience, classic roadmap or augmented visualization to clear ostia and delineate vascular loops.

During catheterization, the second operator took screenshots of the merged images when the wire reached the labeled ROI. These screenshots were used to assess the accuracy of the fusion.

The 3 steps of the workflow are summarized in Fig 2.

\section{Fusion Imaging Assessment}

Spatial accuracy of the MRA/CTA-fluoroscopy fusion roadmap was visually scored using perioperative recorded screenshots and videos.
The operator used a 3-category semiquantitative scale to score the offset: accurate, if the catheter projected within the landmark on the virtual roadmap; mismatch, if the catheter projected out of the virtual landmark but within a distance inferior to the diameter of the vessel; and inaccurate, if the catheter projected out of the landmark with a distance superior to the diameter of the vessel (Fig 3).

\section{Statistical Analysis}

We first calculated the proportion of accurate, mismatch, and inaccurate virtual roadmaps for the 9 ROIs. In a second part, we reunited these ROIs in 2 groups according to their distance from the aortic arch. We defined as close to the arch (group 1) the landmarks labeled on the brachiocephalic artery trunk, the right SCA and vertebral artery, the left common carotid artery, and the left SCA and vertebral artery. We defined as distant from the arch (group 2) the landmarks labeled on the right common carotid artery and ICA, and the left ICA.

AJNR Am J Neuroradiol 41:1663-69 Sep 2020 www.ajnr.org 


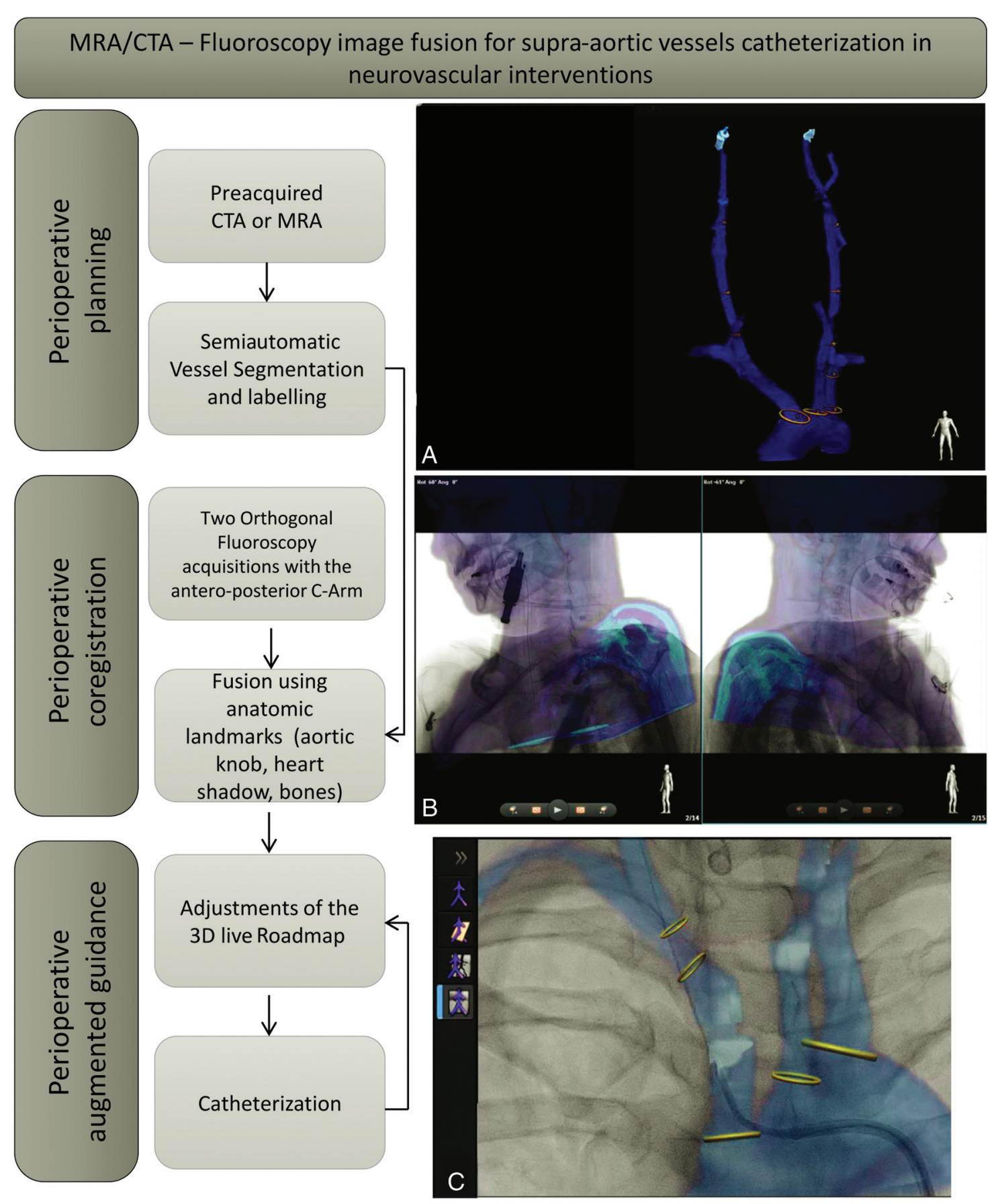

FIG 2. Detailed workflow of the MRA-fluoroscopy or CTA-fluoroscopy image fusion process for supra-aortic vessel catheterization in neurovascular interventions. Preoperative planning starts with uploading the MRA/CTA dataset on VesselNavigator. A, 3D semiautomatic segmentation of the aortic arch and the supra-aortic vessels and ostium labeling. B, Perioperative 2D-3D coregistration with 2 orthogonal positions of the anterior-posterior $C$-arm using anatomic landmarks. $C$, Perioperative augmented guidance.

We compared spatial accuracy between groups 1 and 2 using a mixed-effects logistic regression. A patient random effect was included to take into account the multiple supraaortic vessels analyzed in the same patients. We used the Ime4 package (https://cran.r-project.org/web/packages/lme4/ lme4.pdf) for the mixed-effects model. All statistical analyses were performed using $\mathrm{R}$ statistical and computing software, Version 3.6.1 (http://www.r-project.org/). A $P$ value $<.05$ was considered statistically significant.

\section{RESULTS}

Between May 2019 and October 2019, thirty-three patients (Table 1) underwent a total of 35 sessions of catheterization under MRA/CTA fusion guidance using the VesselNavigator software. Among them, 23 had preprocedual MRA and 12 had preprocedual CTA. A total of 151 supra-aortic vessels were targeted using fusion guidance, from radial access in 4 cases and femoral access in 31 cases. The mean time needed for dataset segmentation and coregistration was $7 \pm 2$ minutes. 

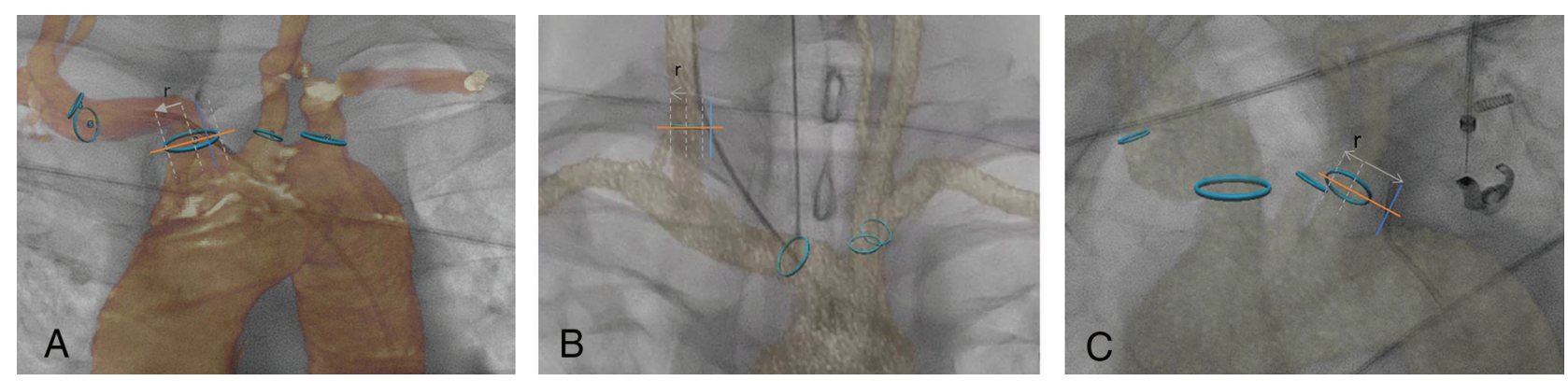

FIG 3. Spatial accuracy assessment of MRA/CTA-fluoroscopy fusion. A 3-category semiquantitative scale was used to measure the maximum offset. $A$, Example of an accurate fusion at the ostium of the brachiocephalic artery trunk: The catheter projects within the lumen of the vessel in the ROI. $B$, Example of a mismatch at the ostium of the right common carotid artery: The catheter projects out of the lumen but within a distance inferior to the diameter of the vessel. $C$, Example of an inaccurate fusion at the ostium of the left common carotid artery: The catheter projects out of the vessel at a distance superior to the diameter.

\begin{tabular}{lc} 
Table 1: Patient characteristics (demographics, cardiovascular \\
risk, procedure features, and aortic arch type) \\
\hline \multicolumn{2}{c}{ Patient Characteristics } \\
\hline Age (yr) \\
Median (Interquartile range) \\
Sex \\
Female (No.) (\%) \\
Male (No.) (\%) \\
Cardiovascular risk factors ${ }^{\text {a }}$ (No.) (\%) \\
0 & $20(61 \%)$ \\
1 & $13(39 \%)$ \\
$\geq 2$ & $12(34 \%)$ \\
Procedure type (No.) (\%) & $6(16 \%)$ \\
Diagnostic/follow-up angiography & $17(50 \%)$ \\
Intracranial embolization & \\
Mechanical thrombectomy & $23(66 \%)$ \\
Anesthesia (No.) (\%) & $9(16 \%)$ \\
Conscious sedation & $3(8 \%)$ \\
General anesthesia & $26(74 \%)$ \\
Noninvasive imaging for navigation (No.) (\%) & $9(26 \%)$ \\
CTA & $12(34 \%)$ \\
MRA & $23(66 \%)$ \\
Aortic arch type (No.) (\%) & \\
I & $20(57 \%)$ \\
II & $8(23 \%)$ \\
III & $7(20 \%)$ \\
Bovine variant & $9(26 \%)$ \\
\hline
\end{tabular}

${ }^{a}$ We considered smoking, high blood pressure, high low-density lipoprotein and low high-density lipoprotein cholesterol levels, age, and obesity.

Semiquantitative assessment showed that $84.8 \%(128 / 151)$ of the virtual landmarks from MRA/CTA overlaid on fluoroscopy were completely accurate, with no need for adjustment or conventional roadmap; $9.9 \%$ (15/151) of the virtual landmarks were overlaid with a mismatch, resulting from vessel deformation caused by endovascular devices; and 5.3\% (8/151) of virtual landmarks were inaccurate, requiring an adjustment or an additional conventional roadmap (Table 2).

Spatial accuracy of the virtual roadmap was higher at the aortic arch level and proximal cervical level (group 1, 92.4\%, 85/92) than at the distal cervical level (group 2, 72.9\%, 43/59). A mixed-effects logistic regression showed a statistically significant difference $(P$ value $=.003)$. The odds ratio was estimated at 4.88 (95\% confidence interval, 1.83-11.66), meaning that the odds of an accurate roadmap were almost 5 times higher in vessels at the aortic arch and proximal cervical level than at the distal cervical level (Table 3: comparison of spatial accuracy between the arch level and proximal cervical level (group 1) and distal cervical level (group 2)).

\section{DISCUSSION}

MRA/CTA fusion guidance for supra-aortic vessel catheterization is feasible in routine clinical practice. The virtual $3 \mathrm{D}$ roadmap offers high spatial accuracy during catheterization, compatible with the safety of neurovascular interventions. Dedicated software such as VesselNavigator allows a fluid workflow, with a duration of $<10$ minutes from MRA/CTA data uploading to 2D$3 \mathrm{D}$ coregistration.

The benefits of augmented navigation provided by image fusion are now recognized for endovascular procedures, in cardiology or aortic endografts. It has been found to reduce procedural time, $\mathrm{x}$-ray exposure, and contrast media. ${ }^{8,10,16-21}$

Planning primary access is a requisite step prior to MRA/ CTA-fluoroscopy fusion. The main benefits are the ability to plan the C-arm position for image acquisition and identify vessel origins and tortuosities on the navigation path. The working angles that accurately identify the targeted vessel ostium might aid catheterization, and they can be stored and recalled during catheterization if needed. The operator could also, at this point, identify the most adapted guidewire or catheter. It is likely that fusion guidance would apply to complex aortic arch catheterizations, especially among the elderly and patients with cardiovascular risk factors. In our study, $25 \%$ of patients were older than 68 years of age, and $50 \%$ had at least 2 cardiovascular risk factors. However, we reported no adverse events related to difficult catheterization (thromboembolic event, dissection, or failure in catheterization).

We used 2D-3D registration, which is faster and easier, especially at the thoracic level. We used 2 orthogonal projections to coregister the segmented 3D-MRA or CTA with live fluoroscopy. A recent work ${ }^{22}$ reported that an accurate localization is possible with 2 projections acquired even at a small $\left(\sim 10^{\circ}\right)$ angular separation. Moreover, 2D registration by fluoroscopic orthogonal shots is a fast, easy technique. The effective $\mathrm{x}$-ray dose is significantly reduced compared with $3 \mathrm{D}-3 \mathrm{D}$ coregistration using conebeam CT with the C-arm. ${ }^{23}$ Few attempts have been made to use MRA/CTA image fusion for neurovascular applications: 3D roadmap navigation is routinely used, and this technique uses 
Table 2: Spatial accuracy assessment of the virtual 3D roadmap for the 9 ROls

\begin{tabular}{|c|c|}
\hline ROls of the Virtual Roadmap/Spatial Accuracy & No. (\%) \\
\hline \multicolumn{2}{|l|}{ Brachiocephalic artery trunk } \\
\hline Accurate & $27(96.4)$ \\
\hline Mismatch & $1(3.6)$ \\
\hline Inaccurate & \\
\hline All & $28(100)$ \\
\hline \multicolumn{2}{|l|}{ Right subclavian artery } \\
\hline Accurate & $8(100)$ \\
\hline Mismatch & \\
\hline Inaccurate & \\
\hline All & $8(100)$ \\
\hline \multicolumn{2}{|l|}{ Right vertebral artery } \\
\hline Accurate & $5(100)$ \\
\hline \multicolumn{2}{|l|}{ Mismatch } \\
\hline \multicolumn{2}{|l|}{ Inaccurate } \\
\hline All & $5(100)$ \\
\hline \multicolumn{2}{|l|}{ Right common carotid artery } \\
\hline Accurate & $16(69.6)$ \\
\hline Mismatch & $3(13.0)$ \\
\hline Inaccurate & $4(17.4)$ \\
\hline All & $23(100)$ \\
\hline \multicolumn{2}{|l|}{ Right internal carotid artery } \\
\hline Accurate & $10(66.7)$ \\
\hline Mismatch & $3(20.0)$ \\
\hline Inaccurate & $2(13.3)$ \\
\hline All & $15(100)$ \\
\hline \multicolumn{2}{|l|}{ Left common carotid artery } \\
\hline Accurate & $24(85.7)$ \\
\hline Mismatch & $3(10.7)$ \\
\hline Inaccurate & $1(3.6)$ \\
\hline All & $28(100)$ \\
\hline \multicolumn{2}{|l|}{ Left internal carotid artery } \\
\hline Accurate & $17(81.0)$ \\
\hline Mismatch & $3(14.3)$ \\
\hline Inaccurate & $1(4.8)$ \\
\hline All & $21(100)$ \\
\hline \multicolumn{2}{|l|}{ Left subclavian artery } \\
\hline Accurate & $13(92.9)$ \\
\hline Mismatch & $1(7.1)$ \\
\hline \multicolumn{2}{|l|}{ Inaccurate } \\
\hline All & $14(100)$ \\
\hline \multicolumn{2}{|l|}{ Left vertebral artery } \\
\hline Accurate & $8(88.9)$ \\
\hline Mismatch & $1(11.1)$ \\
\hline \multicolumn{2}{|l|}{ Inaccurate } \\
\hline All & $9(100)$ \\
\hline \multicolumn{2}{|l|}{ Overall } \\
\hline Accurate & $128(84.8)$ \\
\hline Mismatch & $15(9.9)$ \\
\hline Inaccurate & $8(5.3)$ \\
\hline All & \\
\hline
\end{tabular}

Table 3: Comparison of spatial accuracy between aortic arch and cervical level (group 1) and the distal cervical level (group 2)

\begin{tabular}{|c|c|c|c|}
\hline Spatial Accuracy & $\begin{array}{r}\text { Group } 1 \\
\text { No. (\%) }\end{array}$ & $\begin{array}{r}\text { Group } 2 \\
\text { No. (\%) }\end{array}$ & $\begin{array}{l}\text { Overall } \\
\text { No. (\%) }\end{array}$ \\
\hline Accurate & $85(92.4)$ & $43(72.9)$ & $128(84.8)$ \\
\hline Mismatch/inaccurate & $7(7.6)$ & $16(27.1)$ & $23(15.2)$ \\
\hline
\end{tabular}

image fusion from the same technique. It results in a highly accurate registration ${ }^{11,24-26}$ but is complex to implement at the aortic arch level. Multimodal imaging fusion at the intracranial level is gaining wider acceptance even though reports remain scarce. ${ }^{12,27-29}$ At the aortic arch level, no other reports, to our knowledge, have been published since the pioneering work of Lin et al. ${ }^{15}$

The VesselNavigator image fusion technique was evaluated in 151 supra-aortic vessels, showing a high spatial accuracy for catheterization at the aortic arch and proximal cervical level. However, we observed that the mismatch increased when reaching distal cervical vessels. This limitation has already been reported for abdominal endovascular procedures even with 3D-3D fusion.?

We observed that the patient's head positioning significantly differed on the angiography table from the preoperative MRA/CTA, which resulted in distal cervical vessel distortion. Furthermore, the introduction of stiff guidewires and catheters increased deformation of cervical vessels. As a result, the perioperative vasculature was likely to have a different tridimensional conformation from the MRA/CTA segmented version, leading to fusion mismatch.

Despite the improvement that MRA/CTA fusion brings to catheterization, multiple limitations still prevent its widespread use. Optimizing the workflow with more automated and accurate segmentation and registration processes is necessary. Indeed, these processes are time- and effort-consuming and are considered to interfere with clinical practice, preventing their adoption by physicians. Serious effort in training and dedicated environments might be helpful in the future. ${ }^{30,31}$

The first step that delivers a segmented dataset of the aorta and supra-aortic vessels could be enhanced to provide more accurate datasets for planning the procedure (such as the vessel centerlines, angular information on the vascular path to the target zone, vessel tortuosity index, ostium plane, and so forth).

The benefits of 3D-MRA/CTA fusion guidance might differ according to the type of procedures. For emergency procedures such as patients with stroke, for whom head positioning and agitation will limit distal accuracy of the fusion guidance, great help can be provided to catheterize the proximal supra-aortic trunks in case of complex aortic arch anatomy, which can be challenging in this population. Patients with stroke for whom a diagnostic MRA or CTA has been performed could benefit from fusion guidance with a very moderate increase of procedural time because preprocedural segmentation can be performed before the patient arrives in the angiography suite. Nevertheless, for elective procedures, such as angiograms and aneurysm or AVM treatments, more benefits are expected in terms of reduction of procedural times, $\mathrm{x}$-ray exposure, and contrast medium use. For these patients, adding a preprocedural CTA or MRA only for fusion guidance is questionable and must be further studied but might be beneficial in a selected population for whom more difficult catheterization is expected (especially among the elderly and patients with cardiovascular risk factors).

The registration of the high-resolution 3D model segmented from MRA or CTA should be subdivided according to anatomic landmarks (thoracic, cervical low, cervical high, or intracranial level) to take into account the vasculature distortion related to head position.

Further validations of MRA/CTA fusion techniques in neurovascular interventions will require comparing $2 \mathrm{D}-3 \mathrm{D}$ versus $3 \mathrm{D}-$ $3 \mathrm{D}$ registration in larger studies, at multicentric levels, and using quantitative assessment of the alignment accuracy. 


\section{CONCLUSIONS}

Fluoroscopy with MRA/CTA fusion guidance for supra-aortic vessel interventions is feasible and accurate. Further improvements of the technique to increase accuracy at the distal cervical level and further studies for assessing the procedural time savings and decreasing $\mathrm{x}$-ray radiation exposure for the patient and staff are needed. The potential benefits would apply to complex aortic arch catheterizations, especially among the elderly and patients with cardiovascular risk factors.

Disclosures: Julien S. Savatovsky—UNRELATED: Payment for Lectures Including Service on Speakers Bureaus: Sanofi, Biogen, Philips Healthcare; Travel/ Accommodations/Meeting Expenses Unrelated to Activities Listed: Bayer Healthcare SAS.

\section{REFERENCES}

1. Faggioli GL, Ferri M, Freyrie A, et al. Aortic arch anomalies are associated with increased risk of neurological events in carotid stent procedures. Eur J Vasc Endovasc Surg 2007;33:436-41 CrossRef Medline

2. Werner M, Bausback Y, Bräunlich S, et al. Anatomic variables contributing to a higher periprocedural incidence of stroke and TIA in carotid artery stenting: single center experience of 833 consecutive cases. Catheter Cardiovasc Interv 2012;80:321-28 CrossRef Medline

3. Riga CV, Bicknell CD, Hamady MS, et al. Evaluation of robotic endovascular catheters for arch vessel cannulation. J Vasc Surg 2011;54:799-809 CrossRef Medline

4. Demertzis S, Hurni S, Stalder M, et al. Aortic arch morphometry in living humans. J Anat 2010;217:588-96 CrossRef Medline

5. Yu SC, Leung TW, Hung EH, et al. Angioplasty and stenting for intracranial atherosclerotic stenosis with nitinol stent. Operative Neurosurg 2012;70(1 Suppl Operative):104-13 CrossRef Medline

6. Madhwal S, Rajagopal V, Bhatt DL, et al. Predictors of difficult carotid stenting as determined by aortic arch angiography. J Invasive Cardiol 2008;20:200-04 Medline

7. Goudeketting SR, Heinen SG, van den Heuvel DA, et al. The use of 3D image fusion for percutaneous transluminal angioplasty and stenting of iliac artery obstructions: validation of the technique and systematic review of literature. J Cardiovasc Surg (Torino) 2018;59:26-36 CrossRef Medline

8. Kaladji A, Daoudal A, Clochard E, et al. Interest of fusion imaging and modern navigation tools with hybrid rooms in endovascular aortic procedures. J Cardiovasc Surg (Torino) 2017;58:458-66 CrossRef Medline

9. Kaladji A, Duménil A, Mahé G, et al. Safety and accuracy of endovascular aneurysm repair without pre-operative and intra-operative contrast agent. Eur J Vasc Endovasc Surg 2015;49:255-61 CrossRef Medline

10. Sailer AM, de Haan MW, Peppelenbosch AG, et al. CTA with fluoroscopy image fusion guidance in endovascular complex aortic aneurysm repair. Eur J Vasc Endovasc Surg 2014;47:349-56 CrossRef Medline

11. Blanc R, Seiler A, Robert T, et al. Multimodal angiographic assessment of cerebral arteriovenous malformations: a pilot study. $J$ Neurointerv Surg 2015;7:841-47 CrossRef Medline

12. Kocer N, Kizilkilic O, Babic D, et al. Fused magnetic resonance angiography and 2D fluoroscopic visualization for endovascular intracranial neuronavigation. J Neurosurg 2013;118:1000-02 CrossRef Medline

13. Perhac J, Spaltenstein J, Pereira VM, et al. Improving workflows of neuro-interventional procedures with autostereoscopic $3 \mathrm{D}$ visualization of multi-modality imaging in hybrid interventional suites. Int J Comput Assist Radiol Surg 2016;11:189-96 CrossRef Medline
14. Blanc R, Fahed R, Roux P, et al. Augmented 3D venous navigation for neuroendovascular procedures. J Neurointerv Surg 2018;10:64952 CrossRef Medline

15. Lin CJ, Blanc R, Clarençon F, et al. Overlying fluoroscopy and preacquired CT angiography for road-mapping in cerebral angiography. AJNR Am J Neuroradiol 2010;31:494-95 CrossRef Medline

16. McNally MM, Scali ST, Feezor RJ, et al. Three-dimensional fusion computed tomography decreases radiation exposure, procedure time, and contrast use during fenestrated endovascular aortic repair. J Vasc Surg 2015;61:309-16 CrossRef Medline

17. Tacher V, Lin M, Desgranges $P$, et al. Image guidance for endovascular repair of complex aortic aneurysms: comparison of two-dimensional and three-dimensional angiography and image fusion. J Vasc Interv Radiol 2013;24:1698-1706 CrossRef Medline

18. Kaladji A, Giovannetti M, Pascot R, et al. Preoperative CTscan-based sizing and in-stent restenosis in peripheral endovascular revascularizations. Vascular 2017;25:504-13 CrossRef Medline

19. Klein AJ, Tomkowiak MT, Vigen KK, et al. Multimodality image fusion to guide peripheral artery chronic total arterial occlusion recanalization in a swine carotid artery occlusion model: unblinding the interventionalist. Cathet Cardiovasc Interv 2012;80:1090-98 CrossRef Medline

20. Kobeiter H, Nahum J, Becquemin JP. Zero-contrast thoracic endovascular aortic repair using image fusion. Circulation 2011;124: e280-82 CrossRef Medline

21. Schulz CJ, Schmitt M, Böckler D, et al. Feasibility and accuracy of fusion imaging during thoracic endovascular aortic repair. J Vasc Surg 2016;63:314-22 CrossRef Medline

22. Uneri A, Otake $Y$, Wang AS, et al. 3D-2D registration for surgical guidance: Effect of projection view angles on registration accuracy. Phys Med Biol 2013;59:271-87 CrossRef Medline

23. van den Berg JC. Update on new tools for three-dimensional navigation in endovascular procedures. Aorta (Stamford) 2014;2:279-85 CrossRef Medline

24. Ruijters D, Homan R, Mielekamp P, et al. Validation of 3D multimodality roadmapping in interventional neuroradiology. Phys Med Biol 2011;56:5335-54 CrossRef Medline

25. Soderman $M$, Babic D, Homan $R$, et al. 3D roadmap in neuroangiography: technique and clinical interest. Neuroradiology 2005;47:735-34 CrossRef Medline

26. $\mathrm{Xu} \mathrm{R}$, Leng LZ, Rubin DG, et al. Fusion of intraoperative threedimensional rotational angiography and flat-panel detector computed tomography for cerebrovascular neuronavigation. World Neurosurg 2013;79:504-09 CrossRef Medline

27. Zhang Q, Sun Q, Zhang Y, et al. Three-dimensional image fusion of CTA and angiography for real-time guidance during neurointerventional procedures. J Neurointerv Surg 2017;9:302-06 CrossRef Medline

28. Tritt S, Ommer B, Gehrisch S, et al. Optimization of the surgical approach in AVMs using MRI and 4D DSA fusion technique: a technical note. Clin Neuroradiol 2017;27:443-50 CrossRef Medline

29. Ide S, Hirai T, Morioka M, et al. Usefulness of 3D DSA-MR fusion imaging in the pretreatment evaluation of brain arteriovenous malformations. Acad Radiol 2012;19:1345-52 CrossRef Medline

30. Crossley R, Liebig T, Holtmannspoetter M, et al. Validation studies of virtual reality simulation performance metrics for mechanical thrombectomy in ischemic stroke. J Neurointerv Surg 2019;11:77580 CrossRef Medline

31. Rudarakanchana N, Van Herzeele I, Bicknell CD, et al. Endovascular repair of ruptured abdominal aortic aneurysm: technical and team training in an immersive virtual reality environment. Cardiovasc Intervent Radiol 2014;37:920-27 CrossRef Medline 